Rev. Chil. Padiatr, 62 (5); 309-314, 1991

\title{
Pesquisa etiológica en espasmos masivos
}

\author{
Isabel López $\mathrm{S}^{\perp *}{ }_{;}$Mónica Troncoso Sch. ${ }^{2}$; Ledia Troncoso $\mathrm{A}^{2}$; \\ Marta Colombo C. ${ }^{1}$; Fernando Pinto $\mathrm{L}^{2}$; Oyoni Japaz L. ${ }^{2}$; \\ Fernando Novoa S. ${ }^{2}$
}

\section{An etiologic diagnosis protocol in infantile spasms}

\begin{abstract}
A sistematic clinical protacol was applied in 16 infants that suffered from infantile spasms (IS) in order to identify etiologic factors. A positive family history was present in 2/16 patients and relevant perinatal or postnatal pathology in 5/16. Psvchomotor retardation and other seizures anteceded IS in 10/16 and $8 / 16$ infants respectivelv. Physical and neurologic examination revealed mycrocephalia (4/16), dismorphic features $12 / 16)$, hypopigmented skin lesions $(1 / 16)$ and piramidal syndrome (8/16). Neuroimagine technics $y$ ielded positive findings in 9/16 patients, difuse or localized atrophy (7/16), porencephalic cysts $(3 / 16)$, periventricular calcifications $(1 / 16)$, callosal agenesis $\{1 / 16\}$. Laboratory examination allowed diagnosis of two metabolic diseases: congenital hiperlactatemia an maple syrup urine disease. Two patients were classified as criptogenetic and fourteen as symptomatic. Within the latter an etiologic factor was identified in 12/14. This study underlines the value of etiologic search in IS, because it may contribute substantially to specific treatment and genetic counselling.
\end{abstract}

(Key words: Infantile spasms, etiology.)

Se denomina espasmos masivos (EM) a un síndrome epiléptico grave, propio del lactante, descrito por W. J. West en $1841^{1}$, caracterizado por espasmos breves en salvas. Los pacientes usualmente presentan retardo moderado 0 severo de base y evolucionan con deterioro psicomotor. El trazado electroencefalográfico característico es la hipsarritmia ${ }^{2}$. La incidencia de EM se ha estimado entre 0,24-0,42 x 1000 re. cién nacidos ${ }^{3,4}$ y su etiologia es diversa, dife. renciándose dos grupos de importancia pronóstica: los EM sintomáticos, en que los pacientes tienen alteración neurológica o retarđo psicomotor antes de] inicio de los espasmos o un factor etiológico demostrable por estudios, y los EM criptogenéticos, que ocurren en njños con desarrollo normal y sin antecedentes de enfermedades neurológicas antes de comenzar el cuadro, en los que no se puede identificar un

1. Unidad de Neuropsicología, Instituto de Nutrición y Tecnología de los Alimentos, Universidad de Chile.

2. Servicio de Neuropsiquiatria Infantil, Hospital San Borja-Arriarán.

* Trabajo de Incorporación a la Sociedad de Psiquia. tría y Neurología de la Infancia y Adolescencia, en octubre de 1990. factor etiológico a través del estudio dirigi$\mathrm{do}^{4,5}$. Diferentes enfermedades que producen daño neurológico pueden manifestarse como EM. Su identificación es de gran importancia si se considera que en muchas de ellas esto implica posibjlidades de tratamiento, un aporte al pronóstico del paciente $y$, en algunos casos, un consejo genético para la familia.

En este trabajo nos propusimos desarrollar y aplicar un protocolo de estudio en pacjentes con EM, utilizando los recursos disponibles para precisar la enfermedad subyacente al cuadro convulsivo.

\section{Pacientes y Método}

Los criterios de selección de los pacientes fueron: evidencia clínica de crisis de EM y trazado electroencefalográfico de hipsarritmia típica, modificada o patrones ictales de hipsarritmia según criterios de Gibbs y Hrachovy ${ }^{2}$. La muestra quedó constituida por 16 nifíos, 11 varones y 5 níias, que consultaron al Servicio de Neuropsiquiatría Infantil del Hospital San BotjaAtriarán (ex Paula Jaraquemada) entse julio 1989 y junio 1990, cuyas edades fluctuaron entre 3 y $17 \mathrm{me}$ ses (X: 9,8; d.e.: 4,4 meses) en el momento de ingresar al estudio y entre 2 y 14 meses de edad (X: 5,7 ; d.e.: 3,4 meses) al aparecer los EM, siendo $15 / 16$ casos menores de un ar̃o.

Se desarrolló un protocolo de historia clínica orientado a detectar antecedentes anamnésticos relevantes 
(prenatales, perinatales, exolución psicomotora, ante cedentes familiares) y a efectuar un examen físico y neurológico sistemático. Conjuntamente se desarrolló un esquema de estudio destinado a evaluar el nivel de desarrollo psicomotor, localizar lesiones estructurales, diagnosticar déficit sensoriales e identificar patologia neurológica, metabólic y genética que puede asociarse a EM (tabla 1). Algunos de los proccdimientos se efectuaron en todos los casos, en tanto que otros sốlo cuando el factor etiológico no era claro.

\section{Tabla 1}

Estudio espasmos masivos. Esquema general de tJabajo

A. Antecedentes personales y familiares Historia de enfermedad actual

B. Examen físico y neurológico

- Lámpara de Wood

* Examen neuroftalmológico

* Examen genético

* Examen otorrino

C. Estudios a solicitar:

- Electroencefalograma con piridoxina

- Hemograma

- Pruebas hepáticas

- Electrólitos plasmáticos

- Sedimento urinatio

- TORCH - VDRL

- Rastreo metabólico - aminoaciduria - aminoacidernia

* Líquido cefalorraquídeo: citoquínico - cultivo - cromatograjía de aminoácidos

- pH y gases en sangre arterial, amonemia, lactoacidemia

- USG de cráneo

* TAC cerebro

- Evaluación desarrollo psicomotor. Prueba de Gesel1 ${ }^{\circ}$

* Solamente en caso necesario o posible

\section{Resultados}

Entre los antecedentes anamnésticos de utilidad para orientar al diagnóstico destaca que en 2 casos había hermanos con una enfermedad similar al paciente índice (casos 8 y 12); en otros 2 niños se detectó prematurez, uno de ellos presentó hemorragia intraventricular (caso 4); dos pacientes habian sufrido sepsis neonatal con grave secuela neurológica (casos 9 y 14) y otro niño sufrió un daño anóxico secundario a paro cardiorrespiratorio anestésico (caso 13). Según la información aportada por los padres, $10 / 16$ niños tenían retardo psicomotor antes de inicjar EM. En dos de ellos se señaló clara- mente un deterioro del desarrollo, luego de un período de normalidad. Ocho pacientes presentaron convulsiones de otro tipo antes de comenzar con EM. Por último, un paciente (caso 10) presentó hemorragia cerebral intraparenquimatosa y hematoma subdural derecho, siendo intervenido a los 2 meses de vida. Solamente en 3 casos no hubo alteración neurológica previa (casos 1, 6 y 7) (tabla 2).

El examen físico de estos pacientes demostró que $4 / 16$ presentaban desnutrición severa, con déficit de talla y peso, para la edad según patrones NCHS . En $7 / 16$ casos la circunferencia craneana cayô bajo el percentil 2 para la edad según Tablas de Nellhaus'. El examen de la piel con lámpara de Wood fue positivo en un paciente, demostrando múltiples manchas hipocromas lanceoladas en tronco y extremidades inferjores (caso 6), en tanto que en dos pacientes se observó dismorfias craneofaciales (ca$\operatorname{sos} 2$ y 11) (tabla 2).

En relación al examen neurológico, ocho pacientes presentaron síndrome piramidal bilateral, que en cuatro casos constjtuyó una tetraparesia espástica, en tanto que dos niños presentaron hemiparesia braquiocrural (casos 4 y 10). Un paciente presentó inicialmente hipotonía generalizada, que posteriormente evolucionó hacia un cuadro piramidal bilateral (caso 8).

La evaluación de desarrollo psicomotor efectuada al ingreso demostró un retardo severo en $11 / 16$ casos, moderado en $2 / 15$ casos y leve o normal en 3/15 casos, según prueba de Gesell ${ }^{9}$ (tabla 2). Entre los tres niños con desarrollo normal o compromiso leve se cuentan dos finalmente clasificados como criptogenéticos y un paciente portador de esclerosis tuberosa, que debutó con EM.

En todos los niños se realizó ultrasonografía (USG) cerebral y en cinco tomografía computadorizada (TAC) cerebral. Las técnicas de neuroimagen demostraron lesiones en $9 / 16$ casos, siendo la alteración más frecuente una atrofia difusa (4/8), observada como dilatación del sistema ventricular y aumento de tamaño de los surcos y espacio subaracnoideo. En dos casos se diagnosticó por USG lesiones calificadas como porencefalia (casos 4 y 10) asociadas a atrofia, como secuela de hemorragia intraparenquimatosa (TAC) e infarto silviano, respectivamente (figura 1). Un paciente presentó atrofia temporal bilateral de predominio derecho. La USG demostró lesiones hiperecogénicas periventriculares, en 
Tabla 2

Algu nas características de $\mathbf{1 6}$ pacjentes con espasmos masivos

\begin{tabular}{|c|c|c|c|c|c|}
\hline Caso & $\begin{array}{l}\text { Desarrollo } \\
\text { psiconotor } \\
\text { previo }\end{array}$ & $\begin{array}{l}\text { Crisis } \\
\text { previas }\end{array}$ & $\begin{array}{l}\text { Inicio } \\
\text { espasmos }\end{array}$ & $\begin{array}{l}\text { Lesiones por } \\
\text { neuroimagen } \\
\text { (USG, TAC*) }\end{array}$ & Factor etiológico \\
\hline 1 & Normal & $(-)$ & $14 m$ & $(-)$ & Criptogenético \\
\hline 2 & Ret. moderado & $(+)$ & $3 m$ & $(-)^{*}$ & $\begin{array}{l}\text { Genopatía (hipoacusia, } \\
\text { hipotiroidismo, đismorfias) }\end{array}$ \\
\hline 3 & Deterioro & $(+)$ & $8 \mathrm{~m}$ & $(-)$ & $\begin{array}{l}\text { Enf. orina olor a jarabe dc } \\
\text { arce variante }\end{array}$ \\
\hline 4 & Ret. Leve & $(-)$ & $7 \mathrm{~m}$ & $\begin{array}{l}\text { Infarto silviano } \\
\text { izquierdo*. } \\
\text { Porencefalia }\end{array}$ & $\begin{array}{l}\text { Prematurez, hemorragia } \\
\text { intraventricular }\end{array}$ \\
\hline 5 & Ret. moderado & $(-)$ & $2 \mathrm{~m}$ & $(-)$ & $\begin{array}{l}\text { No precisado, retardo moderado } \\
\text { previo }\end{array}$ \\
\hline 6 & Normal & $(-)$ & $7 \mathrm{~m}$ & $\begin{array}{l}\text { Calcificaciones } \\
\text { periventriculares }\end{array}$ & Esclerosis tuberosa \\
\hline 7 & Normal & $(-)$ & $10 \mathrm{~m}$ & $(-)$ & Criptogenético \\
\hline 8 & Deterioro & $(+)$ & $4 m$ & $\begin{array}{l}\text { Atrofia temporal } \\
\text { bilateral* }\end{array}$ & Hiperlactatemia congénita \\
\hline 9 & Ret. severo & $(+)$ & $3 \mathrm{~m}$ & Atrofia difusa & Sepsis neonatal, microcefalia \\
\hline 10 & Ret. moderado & $(+)$ & $6,5 \mathrm{~m}$ & $\begin{array}{l}\text { Hemorragia intra- } \\
\text { parenquimatosa, } \\
\text { Atrofia parietal } \\
\text { derecha. Porence- } \\
\text { falia derecha }\end{array}$ & Causa no demostrada \\
\hline 11 & Ret. moderado & $(+)$ & $5 \mathrm{~m}$ & $(-)$ & Genopatía inespecifica \\
\hline 12 & Ret. Leve & $(-)$ & $3 \mathrm{~m}$ & Atrofia difusa & Enf. hereditaria no identificada \\
\hline 13 & Ret. severo & $(+)$ & $10 \mathrm{~m}$ & Atrolia difusa & $\begin{array}{l}\text { Dafio anóxico secundario a paro } \\
\text { cardiorrespiratorio anestésico }\end{array}$ \\
\hline 14 & Ret. severo & $(+)$ & $3 \mathrm{~m}$ & Atrofia difusa & Sepsis neonatal \\
\hline 15 & Ret. severo & $(-)$ & $3 \mathrm{~m}$ & $(-)$ & $\begin{array}{l}\text { No precisado, retardo severo, } \\
\text { microcefalia }\end{array}$ \\
\hline 16 & Ret. severo & $(+)$ & $2 \mathrm{~m}$ & $\begin{array}{l}\text { Agenesia cuerpo } \\
\text { calloso. Poren- } \\
\text { cefalia }\end{array}$ & Síndrome de Aicardi \\
\hline
\end{tabular}

USG : Lltrasonografía. TAC: Tomografía axial computadotizada.

un caso (caso 6), y agenesia de cuerpo calloso asociada a quistes aracnoidales (caso 16) (figura 2).

La investigación de laboratorio aportó significativamente al diagnóstico en 2 casos de enfer- medades metabólicas: enfermedad de orina olor a jarabe de arce variante (caso 3) e hiperlactatemia congenjita (caso 8). Por otra parte los exámenes de infecciones congénitas fueron negativos en todos los casos. 


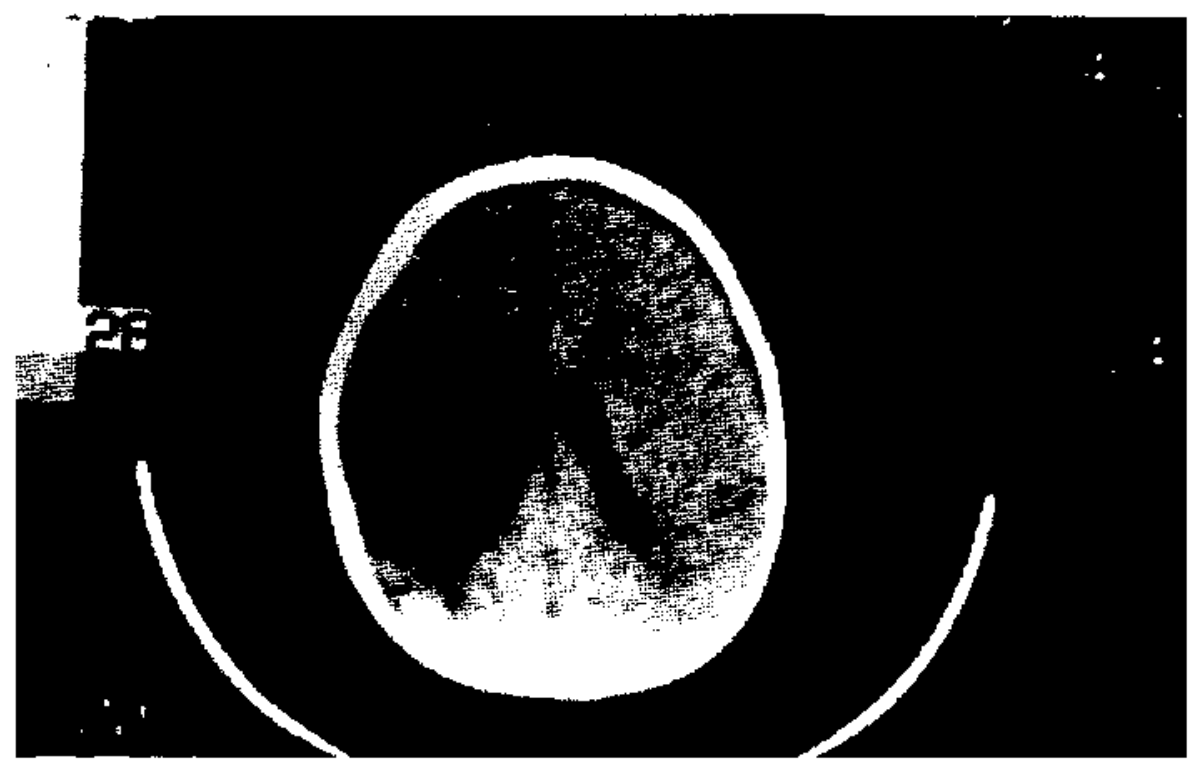

Figura 1: Zona de hipodensidad silviana izquierda, comunicada al ventrículo lateral (caso 4, prematurez, hemorragia intraventricular).

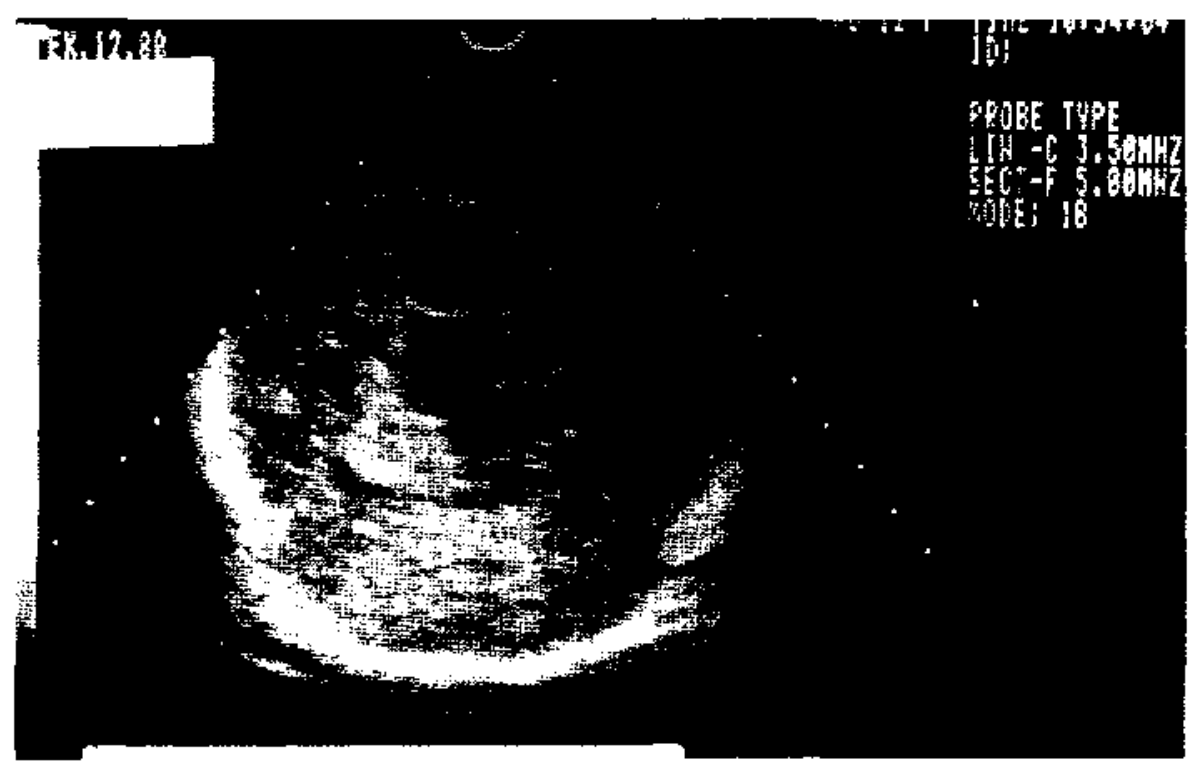

Figura 2: Quiste aracnoidal paraventricular asociado a agenesia de cuerpo calloso (caso 16, Síndrome de Aicardi).

En sintesis. el factor etiológico pudo determinarse en 12/14 niños que conformaron el grupo sintomático; siete de ellos correspondieron a patología prenatal, genética o metabólica, entre los cuales se encuentran errores del metabolismo, dos casos; genopatia, dos casos; esclerosis tuberosa, un caso, y secuelas de afecciones perinatal o postratal, cinco casos. En tres pacientes sintomáticos no fue posible precisar etiología. Dos niños fueron calificados como criptogenéticos, dada su falta de antecedentes patológicos pre. vios sumados a un estudio negativo (casos 1 y 7 ) (tabla 2). 


\section{Comentario}

Tanto la anamnesis como el examen físico aportaron antecedentes relevantes en un número significativo de pacientes. El relato de los padres permitió en muchos casos diferenciar pacientes sintomáticos de criptogenéticos, así como ubicar temporalmente eventos que produjeron una lesión cerebral. Dentro del examen físico destaca la elevada frecuencia de microcefalia y compromiso piramidal, porcentajes similares a lo señalado en otras series $3,5,10$.

El importante retardo en la adquisición de habilidades que presentaba la mayoría de los pacjentes, apunta hacja la gravedad de la alte. ración neurológica. Según señala la literatura, el pequeño subgrupo de tres niños con desarrollo nomal o retardo leve tendría mejores posibjlidades en su pronóstico neurológico a largo pla. zo, que los demás ${ }^{4, ~} \mathbf{1 1}$.

Entre los casos calificados como sintomáti$\cos$ se cuentan dos casos de errores innatos del metabolismo de baja incidencia e improbable djagnóstico si no es con una búsqueda dirigida. Los elementos anamnésticos resultaron orienta. dores en estos pacientes; el antecedente en uno de ellos de un hermano con patología similar y ausencia de patología pre y perinatal que explicara el cuadro y la observación, de parte de los padres, de un período de normalidad en el desarrollo, seguido por un deterioro sin una causa clara. La investigación de laboratorio, que en definitiva acercó al diagnóstico, consideró exámenes que se realizan habitualmente como la búsqueda de aminoácjdos o sus derivados en orina y sangre y la determinación de $\mathrm{pH}$ y gases, amonio y áciđo láctico, pruebas que constituyen una batería minima de investigación ante la sospecha de un error del metabolismo. Los esfuerzos invertidos en el esclarecimiento de estasenfer. medades se tradujeron en medidas específicas de tratamiento de los pacientes (dietas, vitaminas, etc.), así como consejo genético para sus familias.

La USG de cráneo detectó alteraciones en una alta proporción de los casos, siendo ésta una técnica no invasiva y de costo razonable. Cuando se efectuó TAC se obtuvo un diagnóstico lesional en la mayoria de los pacientes. EI porcentaje de lesiones detectado con USG o TAC es algo menor que lo descrito por Gastaut ${ }^{12}$ y Singer ${ }^{13}$, quienes reportan alteraciones tomográficas en 81 y $73 \%$ de sus series, respectiva. mente.
Las lesiones más frecuentes fueron del tipo atrofja global o parcial, luego porencefalia, un caso de calcificaciones periventriculares y un caso de agenesia de cuerpo calloso. Esto es compatible con lo reportado por Gastaut, quien, en un estudio de 37 casos, señala en orden de fre. cuencia: atrofia, malformaciones, calcificaciones, porencefalia y, por último, tumores, en un caso. E1 tipo de malformación descrito con mayor frecuencia en EM es la agenesia de cuerpo calloso como parte de un Síndrome de Aicardi ${ }^{12,14}$, lesión de fácil identificación con USG como ocu. rrió en el caso 16 de esta serie. No ocurre lo mismo con el diagnóstico de tumores de SNC, que requiere de TAC cerebral de buena resolu. ción. No obstante, las lesiones tumorales son una causa rara de EM. Su diagnóstico es de la mayor importancia, puesto que constituyen afecciones even tualmente tratables con modificación sustancial en el pronóstico del paciente ${ }^{15}$. Tanto los resultados de este trabajo como de otras series estudiadas enfatizan la importancia de utilizar de rutina en pacientes con EM técnicas de neurojmagen no invasjvas, como TAC, o eventualmente resonancia magnética, con e] objeto de identificar lesiones cerebrales, evaluar su severidad, aportar a diagnósticos específicos y eventualmente proponer tratamientos.

Por último, se diagnosticó un caso de esclerosis tuberosa, sindrome neurocutáneo, de herencia dominante, que con frecuencia se asocia a EM.

Bajo el diagnóstico sindromático de EM se agrupa una gran diversidad de factores etiológicos que dañan al SNC. Estos pueden identificarse en un elevado porcentaje de pacientes, a través de la aplicación de un protocolo de estudio sistemático, como el que se propone en este trabajo. Siendo este un síndrome convulsjoo que generalmente implica un pronóstico pobre, el reconocimiento oportuno de su calusa puede significar tratamiento especifico y consejo genético, así como aportar en parte al pronóstico del paciente

\section{Resumen}

Con el objeto de identificar un factor etiológico se aplicó un protocolo de estudjo sistemático en 16 lactantes que presentaban espasmos masivos. En dos pacientes hubo antecedentes familiares relevantes, en tanto que cinco 
presentaron afecciones perinatales o postnatales graves. Diez niños presentaron retardo psicomotor y ocho otras crisis previo al inicio de EM. E1 examen físico reveló microcefalia ${ }^{4}$, dismorfias $^{2}$, manchas hipopigmentadas de la piel ${ }^{1}$, síndrome piramidal ${ }^{8}$. Las técnicas de neuroimagen demostraron hallazgos positivos en 9 casos, atrofia en 7 , porencefalia en 3 , calcificaciones en uno y agenesia del cuerpo calloso en uno. El laboratorio permitió el diagnóstico de dos casos con enfermedades metabólicas: hiperlactatemia y enfermedad de orina olor a jarabe de arce. Dos pacientes se catalogaron como criptogenéticos y 14 como sintomáticos. Entre los últimos en doce casos se identificó razonablemente una etiología. Este estudio enfatiza el valor de la búsqueda etiológica en EM, puesto que aporta al tratamiento específico y/o consejo genético en algunos pacientes.

(Palabras clave: Espasmos masivos, infantiles, etiología.)

\section{Agradecimientos}

Agradecemos al personal de enfermeria del Servicio de Neuropsiquiatría Infantil del Hospital San Borja por su colaboración en la realización de este trabajo.

\section{Referencias}

1. West WJ: On a peculiar form of jnfantile convulsions. Lancet 1841; 1: 724-725.

2. Gibbs FA, Gibbs EL: Atlas of electroencephalography. Vol. II: Epilepsy. Addison-Wesley, Cambridge, Mass, 1952; 24-25.
3. Riikonen $R$, Doner $M$ : Incidence and etiology of infantile spasms from 1960 to 1976. A population study in Findland. Dey Med Child Neurol 1979; $21: 333-343$.

4. Rikonen $R$ : Infantile spasms: Modern practical aspects. Acta Pediatr Scand 1984; 73:1-12.

5. Jeavons PM: West syrdrome: Infantile spasms. En: Epileptic syndromes in infancy, childhood and adolescence. Eds. J Roger, C Dravet, M Bureau, FE Dreifuss, P Wolf. John Libbey. Eurotext. London. $1985 ; 42-50$.

6. Hrochovy RA, Frost JD, Kellaway P- Hypsarrhytmia: Variations on the theme. Epilepsia 1984; $25: 317-325$.

7. National Center for Health Statistics. Growth curves for children: Birth-1B years, Charts. US Department of Health Education and Welfare Public Health Service, DHEW 1977, Vital and Health Statistics Series 11, Number 165, 1977.

8. Nellhaus $G_{\text {; }}$ Head circumference from birth to eighteen years. Pediatrics $1986 ; 41: 106$.

9. Gesell $A$ y Amatruda C: Diagnóstico del desarro1lo. Ed. Paidós, Buenos Aires, 1970.

10. Matsumoto $A$, Watanabe $K$, Negoro $T$ et al.: Longterm prognosis after infantile spasms: a statistical study of ptognostic factors in 200 cases. Dev Med Child Neurol 1981;23:51-65.

11. Aicardi $J$ : Treatment of infantile spasms. En: Intractable epilepsy. Eds. D Schmidt and PL Morselli. Raven Press. N.Y. 1986: 147-156.

12. Gastaut H, Gastaut JL, Régis $H$ et al. : Computarized tomography in the study of West Syndrome. Dev Med Child Neurol 1978; 20: 21-27.

13. Singer WD, Haller JS, Sultivan $L R$, Wolpert $S$, Mills $C_{1}$ Rabe EF: The valuc of neuroradiology in infantile spasms. J Pediatr 1982; 100: 47-50.

14. Aicardi $K$, Lefevre $K$, Lerique-Koechlin $A$ : А nеw syndrome: spasms in flexion, callosal agenesis, ocular abnormalities. Electroencephalog Clin Neurophysiol $1965 ; 19: 609-610$.

15. Ruggieri $V$, Caballa $R$, Fejeman $N$ : Intracranial tumors and West synd rome. Pediatr Neurol 1989; $5: 32\}-329$.

\section{AVISO A LOS AUTORES}

Por acuerdo del Comité Editorial, la Revista Chilena de Pediatria devolverá sin tramitar todos los trabajos que no den estricto cumplimiento al Reglamento de Publicaciones, que se edita en cada número, y a las Instrucciones a los Autores, que aparecen en los números 3 y 6 de cada volumen desde 1986. 